\title{
Quantum Automated Object Detection Algorithm
}

\author{
Ling $\mathrm{Hu}$, Qiang $\mathrm{Ni}$ \\ School of Computing and Communications, \\ Lancaster University, InfoLab21, \\ Lancaster, UK, LA1 4WA \\ E-mail:\{1.hu, q.ni\}@lancaster.ac.uk
}

\begin{abstract}
Automated object detection algorithm is an important research challenge in many applications such as computer vision and urban surveillance systems. Recently, quantum computing and information processing become emerging technologies which attract researchers' increasing attention and some work has been attempted in image detection using quantum computing approach. In this paper, we propose a novel quantum automated object detection algorithm for urban surveillance systems. This novel quantum based method is shown to be capable of detecting an object in images accurately and is able to deal with the measurement errors caused by the quantum measurements in the image retrieving process.
\end{abstract}

Keywords- Automated object detection; urban surveillance systems; quantum image; quantum computing

\section{INTRODUCTION}

In recent years, the research of quantum computing and quantum information processing become more and more inspiring [1]. Quantum computers make use of quantum mechanical phenomena to perform operations on data; they can solve many problems which are difficult for normal computers [2]. Specially, a growing number of researchers make efforts on quantum image processing (QIP) since computer vision is one most important channel of obtaining information [3].

Different from traditional methods such as histogram statistics based image analysis [4], several QIP methods were proposed. In [5], the authors proposed an initial step of QIP. This method uses qubits to store colours of an image, and then store the image in a qubit lattice. The authors in [6] proposed a method to store and retrieve images in entangled quantum systems, which uses some 2qubits and 3-qubits entangled quantum states to store images. However, both methods need a certain number of measurements for the retrieving process, which make the computational complexity high. Another unavoidable problem is that the quantum process can only be measured once. After the only once measurement, the quantum state will collapse to a fixed state with some probability [7], which makes the following experiments meaningless.

The authors in [8] proposed a Flexible Representation of Quantum Images (FRQI) method to provide the colours and their corresponding positons in an image on quantum computers. In [9], FRQI was improved and an enhanced quantum representation (NEQR) for digital images was proposed, which uses the basis state of a qubit sequence to store the grey-scale value of each pixel in the image. The authors in [10] encode the pixel values of the image in the probability amplitudes and the pixel positions in the computational basis states. In [11], a quantum image representation based on bit planes (BRQI) is proposed, which represents a grayscale and colour image of $2 \mathrm{n}$ pixels using $(n+4)$ and $(n+6)$ qubits, respectively. However, all these proposed methods did not consider the probability errors caused by the measurements in the image retrieving process which may reduce the object detection accuracy significantly in these methods.

\section{OUR QUANTUM IMAGE SySTEM}

In practical applications, such as urban surveillance applications, automated object detection is an important task [12] [13]. Due to the huge amounts of images, images are normally converted to grey image form to reduce the size of the files. Hence, in this research we will store and process the grey images in quantum systems. 1-qubit quantum computing system is used.

A grey image is constituted by $m \times n$ pixels, and every pixel can be represented by a grey scale value $G(x, y)$ $(0 \leq \mathrm{G}(\mathrm{x}, \mathrm{y}) \leq 1)$. Let us define $Q$ as the corresponding qubit lattice, and denote $T(x, y)=\sqrt{G(x, y)}$, then

$$
\begin{aligned}
& Q=\{\mid q(x, y)>\}, \\
& \text { where } 0<x<m, 0<y<n
\end{aligned}
$$

For every qubit pixel,

$$
\begin{aligned}
& |q(x, y)>=\cos \theta(x, y)| 0>+\sin \theta(x, y) \mid 1>, \\
& \text { where } \cos \theta \quad(x, y)=T(x, y)
\end{aligned}
$$

In this way, we successfully load the grey value of every pixel into the quantum qubit pixels lattice.

We know that the observation of a quantum state is a probability process. As mentioned in [6], when we measure pixels of an image, the quantum states will collapse to certain states. The probability of quantum collapse to state $\mid 0>$ is:

$$
\begin{aligned}
P(x, y)(\mid 0>) & =\cos \theta(x, y) \times \cos \theta(x, y) \\
= & T(x, y) \times T(x, y) \\
= & G(x, y)
\end{aligned}
$$

It is known that the measurement can only be done once and the quantum will collapse to basis $\mid 0>$ with the probability of grey scale value of every pixel. After this retrieving process, the quantum states will stay there unchanged not matter how many further measurements will be done. In this way, the retrieved image will not be accurate. For each pixel, the observation process results in measurement errors, and this type of errors cannot be solved by multiple measurements as the same measurements cannot be repeated due to quantum collapse. 


\section{Quantum Automated ObJect Detection ALGORITHM}

It is known that the observation process results in measurement errors for quantum images, but the same kind of measurements could only be done once, hence multiple measurements are useless. In this paper, we propose a novel method to deal with this challenging problem: We propose to calculate different pixels in the image to determine our object parameters, since the measurement errors will be cancelled by the mean value of various pixels. Considering the character of quantum image, we will implement our method to detect the main object area of an image, by using the retrieved quantum image with the collapsed noise.

We will use an example image to illustrate how our proposed method works. Figure 1 is a grey image taken from urban surveillance systems, there is a car inside this image. Inside this image, every pixel represents its grey value which is between 0 and 1 . We load it into the quantum system using our image storage method. After retrieving this image from the quantum system, measurement errors will occur according to the character of quantum systems' observation process. Our aim is to detect this car with our proposed method.

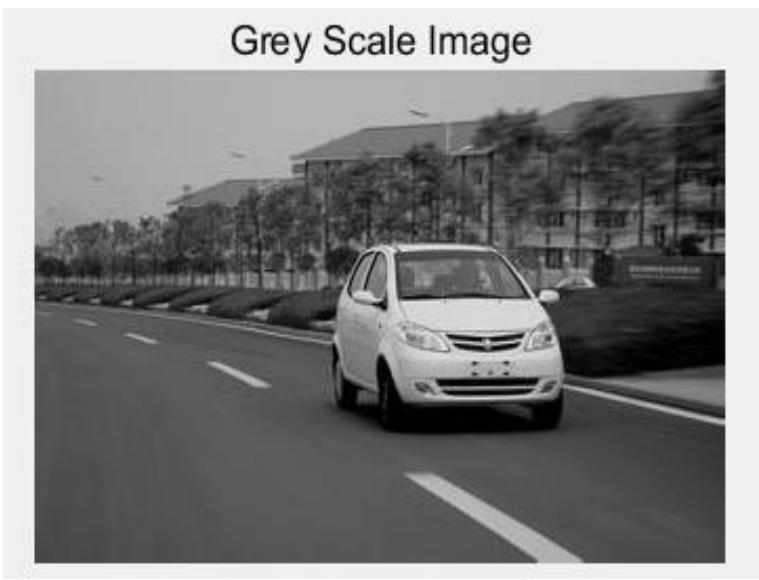

Figure 1. Grey image of a car

The first step is to obtain the gradient of the grey image, which means to calculate the numerical gradient of the image matrix. We define $P_{x}$ to correspond to the differences in $\mathrm{x}$ (horizontal) direction and $P_{y}$ to correspond to the differences in y (vertical) direction. The definitions of $P_{x}$ and $P_{y}$ are:

$$
\left\{\begin{array}{l}
P_{x(i, j)}=I(i+1, j)-I(i, j) \\
P_{y(i, j)}=I(i, j+1)-I(i, j)
\end{array},\right.
$$

Where $I$ is the grey scale value of the pixels when $(i, j)$ is the position of the pixel.

For every pixel, after getting the gradient of both the horizontal and vertical directions, we calculate the overall gradient for this pixel:

$$
P=\sqrt{P_{x}^{2}+P_{y}^{2}}
$$

Figure 2 shows the calculated gradient of the original grey image.

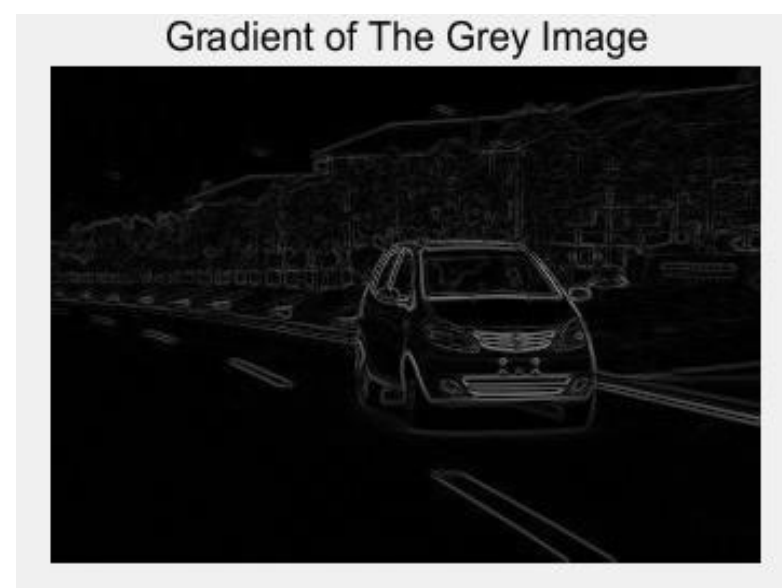

Figure 2. Gradient of the grey image

Then we add every column's values to create a new row numbers, which shows the $\mathrm{x}$-axis brightness of the gradient image. After normalization, we plot the new row in Figure 3. At the high frequency areas, this brightness values are high since there are more changes in original grey image.

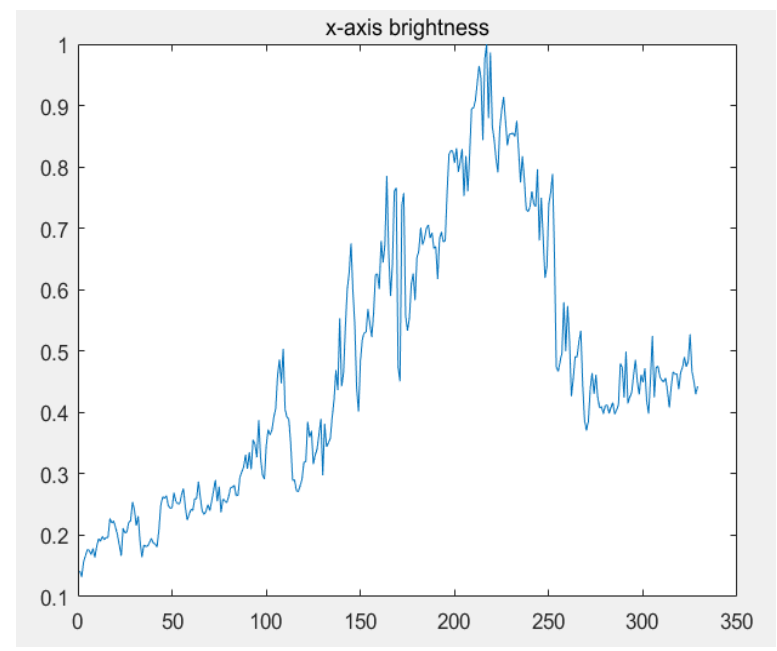

Figure 3. X-axis brightness

The numbers in Figure 3 are not suitable for our judgement since there are a lot of vibrations inside the figure. We smooth the values by calculating their neighbours' mean values (13 neighbours) and the smoothed values are plotted in Figure 4. We only simply choose the threshold at 0.5 , the car area in $\mathrm{X}$-axis is picked out. 


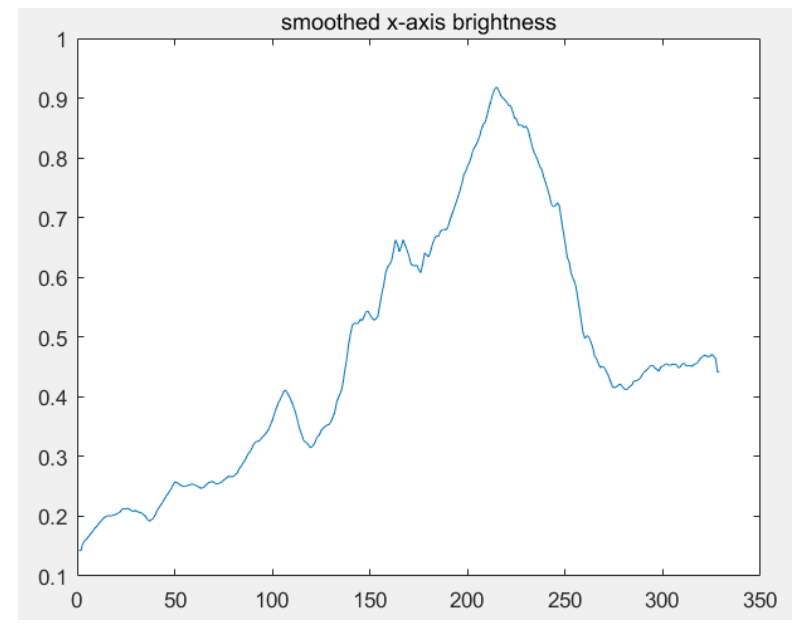

Figure 4. Smoothed $\mathrm{x}$-axis brightness

Using the same method on the y-axis, we get $y$-axis brightness values shown in Figure 5. Because the image content in $y$-axis changes more significantly than in $\mathrm{x}$ axis, we need to average with more neighboring pixels in $\mathrm{y}$-axis, which is chosen as 33 . The smoothed values are shown in Figure 6.

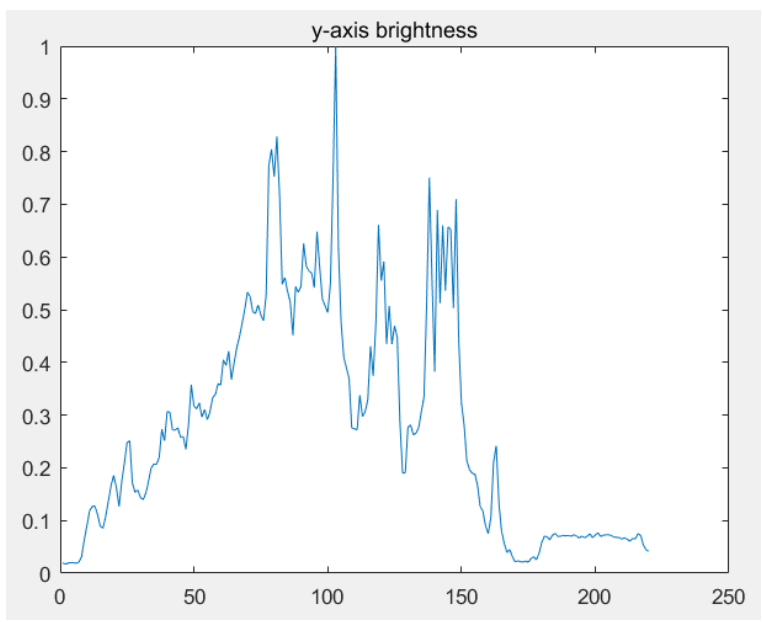

Figure 5. Y-axis brightness

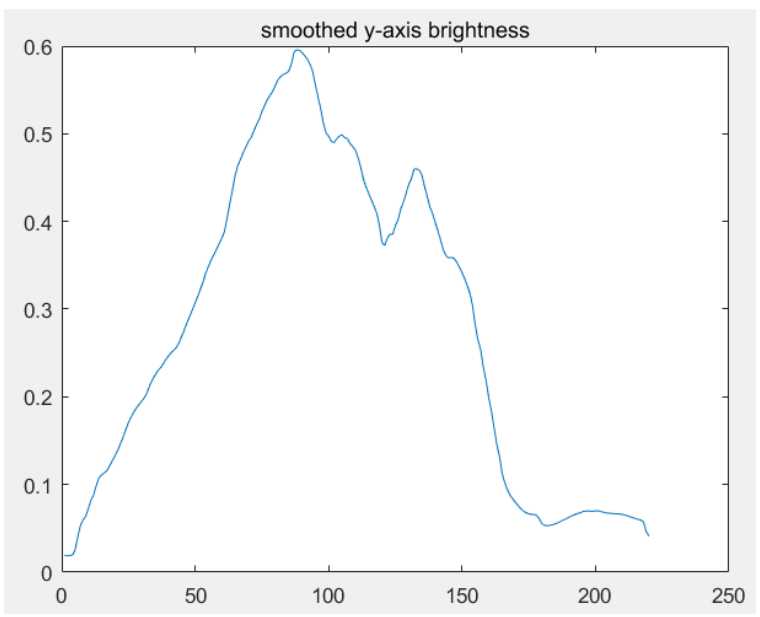

Figure 6. Smoothed y-axis brightness
By choosing appropriate threshold (0.3), the car area in y-axis is also picked out by us. Figure 7 shows the detected area; we can find the car inside this area.

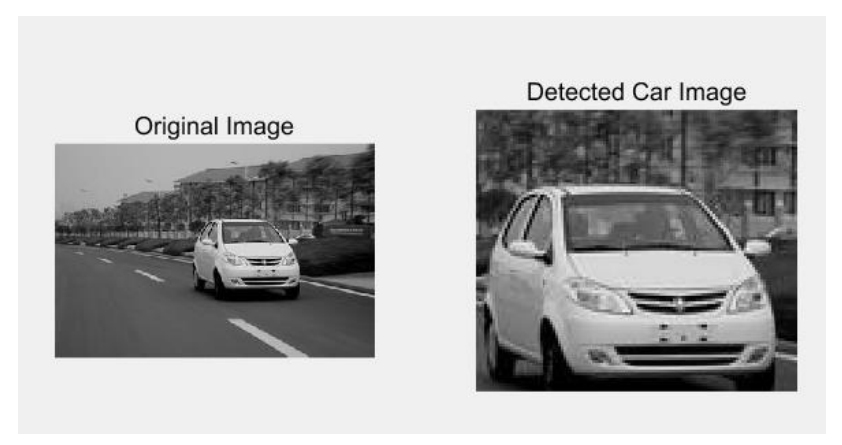

Figure 7. The detected car area

Since our method is able to find out the highest energy area in an image, it can also be used to find a vehicle license plate in vehicle images. Below is an example.

Figure 8 is another car grey image and our objective is to filter out the car license plate.

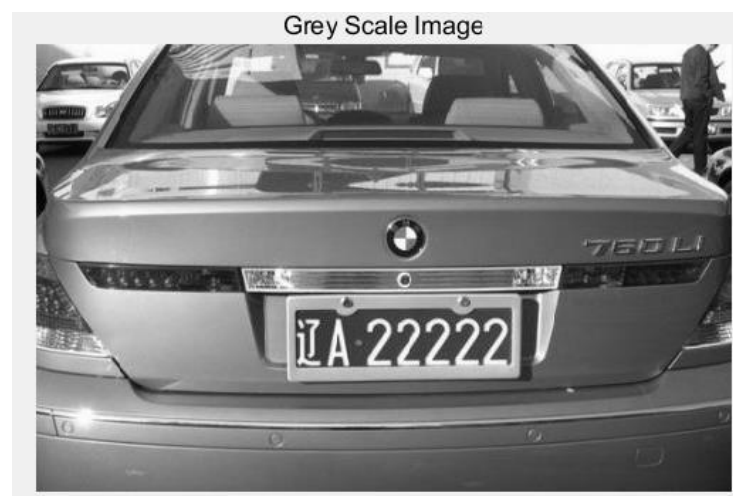

Figure 8. Grey image of another car

Using exactly the same method, the smoothed $\mathrm{x}$-axis brightness values are shown in figure 9. Figure 10 shows the $y$-axis brightness values.

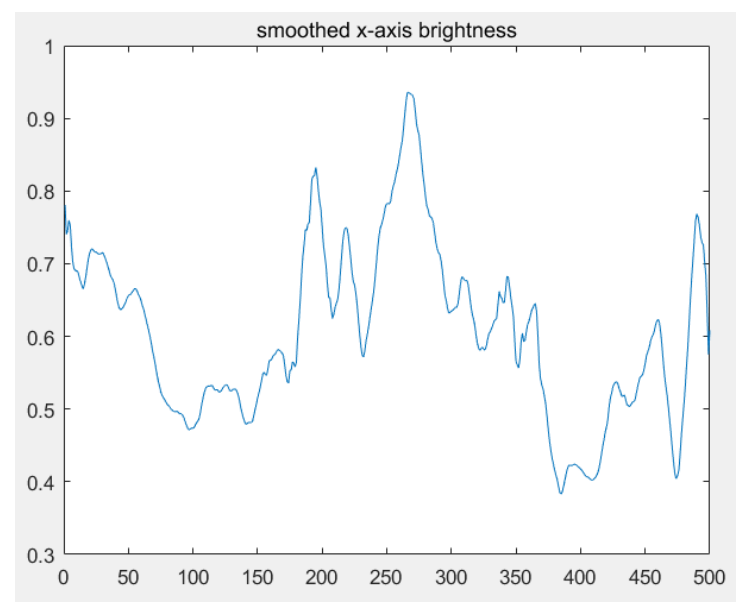

Figure 9. Smoothed $\mathrm{x}$-axis brightness 


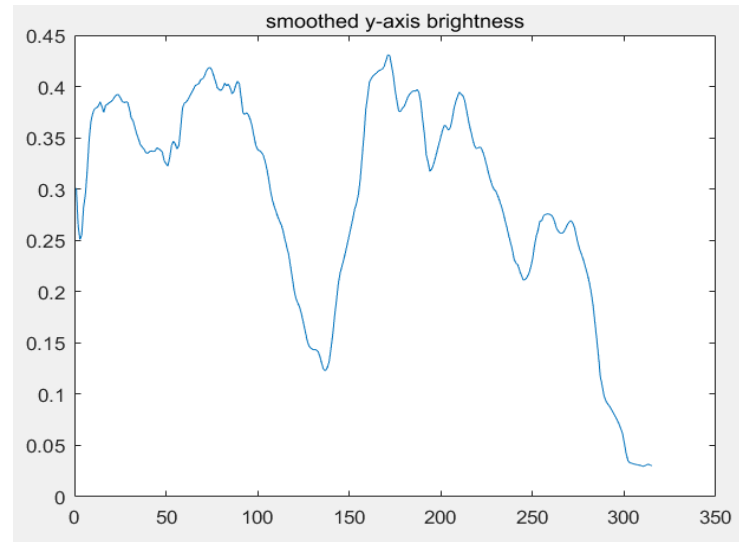

Figure 10. Smoothed y-axis brightness

By choosing same thresholds, the car plate is also picked out by us. Figure 11 shows the detected area; the car plate is detected.

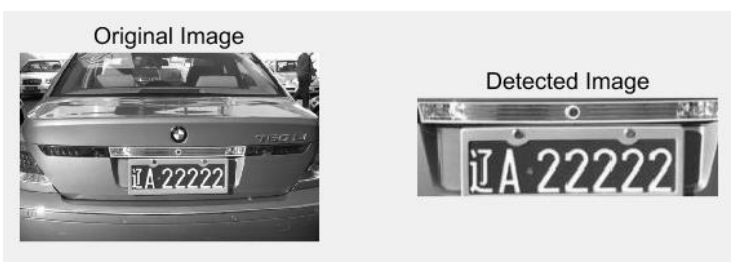

Figure 11. The detected plate area

Within our method, the process of calculating the gradient of the grey image used subtraction operation; Xaxis and y-axis brightness calculation used addition operation; the smoothing process used mean values of several pixels. All these calculations reduced the measurement errors caused by the observation step (i.e. the measurement process) of quantum systems.

\section{CONCLUSION}

This paper presents a new quantum automated image object detection method to automatically detect object (e.g. vehicle or vehicle plate) in urban surveillance systems. To mitigate the impact of the quantum measurement errors, we proposed to calculate the mean value of different pixels in the image from each quantum measurement considering that the same quantum measurement could only be done once due to the quantum collapse characteristic. The simulation results demonstrate that our method can accurately detect the vehicle or vehicle plate from the images.

\section{REFERENCES}

[1] D. Castelvecchi, "Quantum computers ready to leap out of the lab in 2017", Nature, Vol. 541, pp. 9-10, January 2017.

[2] K. Rao, "Computer systems architecture vs quantum computer", 2017 International Conference on Intelligent Computing and Control Systems (ICICCS), pp. 1018 $1023,2017$.

[3] Y. Cai, X. Lu, N. Jiang, "A survey on quantum image processing", Chinese Journal of Electronics, Volume: 27 , Issue: 4, pp. 718-727, 2018.

[4] Z. Huo, Y. Zhang, R. Sath, L. Shu, "Self-adaptive fault diagnosis of roller bearings using infrared thermal images", IECON 2017 - 43rd Annual Conference of the IEEE Industrial Electronics Society, 2017.

[5] S.E.Venegas-andraca and S.Bose, "Storing, processing and retrieving an image using quantum mechanics", Proc.SPIE Conf. Quamtum Inf.Comput. 5105, pp. 137-147, 2003.

[6] H. Li, Q. Zhu, R. Zhou, Y. Pu, "Image storage, retrieval and compression in entangled quantum systems", 2014 IEEE International Conference on Progress in Informatics and Computing, pp. $237-241,2014$.

[7] M. Berta, J. M. Renes, M. M. Wilde, "Identify the information gain of a quantum mesurement", IEEE Transactions on Information Theory, Vol. 60, Issue 12, pp. 7987 - 8006, December 2014.

[8] P. Quang, F. Dong, Y. Arai and K. Hirota, "Flexible representation of quantum images and its computational complexity analysis", 25th Fuzzy System Symposium, 2009 Japan Society for Fuzzy Theory and Intelligent Informatics.

[9] Y. Zhang, K. Lu, Y. Gao, and M. Wang, "NEQR: a novel enhanced quantum representation of digital images", Quantum Information Processing, Vol. 12, Issue 8, pp. 2833-2860, August 2013.

[10] X.W. Yao, H. Wang, Z. Liao, M. Chen, J. Pan, J. Li, K. Zhang, X. Lin, Z. Wang, Z. Luo, W. Zheng, J. Li, M. Zhao, X. Peng, D. Suter, "Quantum image processing and its application to edge detection: theory and experiment", Physical Review X 7, 031041, 2017.

[11] H. Li. X. Chen, H. Xia, Y. Liang, Z. Zhou, "A quantum image representation based on bitplanes", IEEE Access, Vol. 6, pp. 62396 - 62404, Spetember 2018.

[12] L. Hu, Q. Ni, "IoT-driven automated object detection algorithm for urban surveillance systems in smart cities", IEEE Internet of Things Journal, Vol. 5, Issue 2, pp. 747 754, April 2018.

[13] L. Hu, Q. Ni, F. Yuan. "Big data oriented novel background subtraction algorithm for urban surveillance systems", Big Data Mining and Analytics Journal, Vol. 1, No. 2, pp. 137-145, June 2018. 